\title{
ТОРМОЗНАЯ ЭФФЕКТИВНОСТЬ ПОЕЗДА МЕТРО С ДИСКОВЫМИ ТОРМОЗАМИ ПРИ ЮЗОВОЙ СИТУАЦИИ ВRАКЕ
}

\author{
PERFORMANCE SUBWAY TRAIN \\ WITH DISC BRAKES SITUATION IN UNIONS
}

\author{
Представил д-р техн. наук, профессор И.Э. Мартынов
}

Введение. Приоритетными задачами развития коммунального транспорта и, в частности, метрополитена, являются улучшение транспортного обслуживания населения, повышение комфортности, а также увеличение объема пассажирских перевозок путем повышения скорости движения поездов, обусловленное применением более эффективных тормозных систем.

Для удовлетворения этих задач на ПАТ «КВСЗ» созданы вагоны метро нового поколения, отличительной особенностью которых является наличие системы пневмоподвешивания и тормозной системы, оборудованной дисковыми тормозами с противоюзными устройствами, на которых была проведена экспериментальная часть исследований.

Постановка проблемы. Важнейшим показателем тормозной эффективности поезда метро является тормозной путь, так как остановка поезда должна происходить строго в пределах станционной платформы для высадки и посадки пассажиров. Величина тормозного пути зависит от множества факторов, одним из которых является коэффициент сцепления колеса с рельсом. Если в тоннелях коэффициент сцепления сохраняет стабильные значения, то на открытых участках его величина зависит от погодных условий. Такие факторы, как дождь, мокрый снег, обледенение, туман, и прочие, приводят к уменьшению коэффициента сцепления колеса с рельсом и при определенных условиях к юзу колесной пары.

Дисковые тормозные системы обладают более высокой тормозной эффективностью по сравнению с колодочными тормозами. Повышенное значение удельной тормозной силы увеличивает вероятность ее превышения над коэффициентом сцепления колеса с рельсом, а значит, и возможность возникновения юзовой ситуации и блокировки колесных пар при торможении. Наличие противоюзных устройств предотвращает полную блокировку колесных пар путем отпуска тормозов на колесной паре, попавшей в юзовую ситуацию. При достижении колесной парой частоты вращения, соответствующей линейной скорости движения вагона, тормозные цилиндры вновь наполняются сжатым воздухом.

Противоюзные устройства служат для предотвращения блокировки колесных пар и уменьшают вероятность повреждения колесных пар при торможении, однако тормозной путь поезда при этом увеличивается, поэтому одной из актуальных становится задача оценки тормозной эффективности поезда метро при срабатывании противоюзных устройств. 
Изложение материала. Экспериментальные исследования проводились на открытом участке пути «Днепр-ЛеснаяДнепр» Киевского метрополитена как при благоприятных, так и при неблагоприятных погодных условиях (мелкий дождь, туман, мокрый снег), гарантирующих низкий коэффициент сцепления колеса с рельсом.

Тормозная эффективность поезда метро при действии противоюзного устройства оценивалась по величине тормозного пути для скоростей движения 40-80 км/ч.

В качестве датчиков оборотов использовались штатные датчики противоюзных устройств, расположенные в торцевой части оси колесных пар.

Накопленный опыт ходовых тормозных испытаний единиц подвижного состава железных дорог свидетельствует, что модель тормозного пути может быть представлена полиноминальной зависимостью от скорости в начале торможения:

$$
S=\sum_{k=1}^{m} a_{k} \cdot V^{k}
$$

где $S$ - тормозной путь, м;

$V$ - скорость в начале торможения, м/с;

$m$ - порядок (число членов) степенного ряда (1);

$a_{k}$ - коэффициенты ряда (1), подлежащие определению.

Выбор модели торможения производится по минимальному значению коэффициента детерминации и остаточной дисперсии:

$$
S=\sum_{k=1}^{m} a_{k} \cdot V^{k}+u
$$

где $u$ учитывает наличие влияния факторов, которые не входят в заданную модель.

Уравнение (2) описывает зависимость фактических значений фактора $S$ для фактических значений независимой переменной $V$. Расчетные значения $\widehat{S}$ описываются уравнением

$$
\widehat{S}=\sum_{k=1}^{m} \widehat{a}_{k} \cdot V^{k}
$$

Чтобы уравнение (3) адекватно описывало фактическую зависимость $S$ от $V$, необходимо определить такие параметры $\hat{a}_{k}$, при которых отклонения фактических значений $S$ от расчетных $\widehat{S}$ были бы минимальными. В этом случае минимизации подвергается сумма квадратов отклонений (остатков) [1]:

$$
\left\{\sum_{i=1}^{n} u_{i}^{2}\right\}=\min ,
$$

где $u=S-\widehat{S}$.

Для определения коэффициентов $\hat{a}_{k}$ уравнения (3) используется метод максимального правдоподобия (метод наименьших квадратов).

Анализ многочисленных экспериментальных исследований тормозной эффективности поездов метро свидетельствует, что для адекватного отображения тормозного пути достаточно ограничиться квадратичной степенной зависимостью.

Выбор степенной зависимости производится по максимальному значению коэффициента детерминации и остаточной дисперсии.

Система разрешающих уравнений определяется выражением для полинома второй степени [1]:

$$
U=\sum_{i=1}^{n}\left(S_{i}-\left(a_{2} \cdot V_{i}^{2}+a_{1} \cdot V_{i}\right)\right)^{2},
$$

где $\mathrm{n}$ - число интервалов исследуемого диапазона скоростей.

Определяя частные производные функций (5) по коэффициентам $a_{i}$ и приравнивая их к нулю, получим: 


$$
\left\{\begin{array}{l}
\frac{d U}{d a}=\sum_{i=1}^{n}\left(S_{i}-\left(a V_{i}^{2}+b V_{i}\right)\right) \cdot V_{i}^{2}=0 \\
\frac{d U}{d b}=\sum_{i=1}^{n}\left(S_{i}-\left(a V_{i}^{2}+b V_{i}\right)\right) \cdot V_{i}=0
\end{array} .\right.
$$

Окончательно полученную систему уравнений (6), получим:

$$
\left\{\begin{array}{l}
a_{2} \sum_{i} V_{i}^{4}+a_{1} \sum_{i} V_{i}^{3}=\sum_{i} S_{i} V_{i}^{2} \\
a_{2} \sum_{i} V_{i}^{3}+a_{1} \sum_{i} V_{i}^{2}=\sum_{i} S_{i} V_{i}
\end{array},\right.
$$

решая которую найдем:

$$
\begin{aligned}
& a_{2}=\frac{\sum_{i=1}^{n} S_{i} V_{i}^{2} \sum_{i=1}^{n} V_{i}^{2}-\sum_{i=1}^{n} S_{i} V_{i} \sum_{i=1}^{n} V_{i}^{3}}{\sum_{i=1}^{n} V_{i}^{4} \sum_{i}^{n} V_{i}^{2}-\left(\sum_{i=1}^{n} V_{i}^{3}\right)^{2}}, \\
& a_{1}=\frac{\sum_{i=1}^{n} S_{i} V_{i} \sum_{i=1}^{n} V_{i}^{4}-\sum_{i=1}^{n} S_{i} V_{I}^{2} \sum_{i=1}^{n} V_{i}^{3}}{\sum_{i=1}^{n} V_{i}^{4} \sum_{i}^{n} V_{i}^{2}-\left(\sum_{i=1}^{n} V_{i}^{3}\right)^{2}} .
\end{aligned}
$$

Коэффициент детерминации $R^{2}$ определяется по формуле [1]

$$
R^{2}=1-\frac{\sum_{i=1}^{n}\left(S_{i}-\left(a_{2} \cdot V_{i}^{2}+a_{1} \cdot V_{i}\right)\right)^{2}}{\sum_{i=1}^{n} S_{i}^{2}-\frac{\left(\sum_{i}^{n} S_{i}\right)^{2}}{n}} .
$$

Отсев грубых погрешностей осуществляется статистическими методами при уровне значимости $\mathrm{p}=0,05$, который соответствует доверительной вероятности 0,95. Для этого определялась абсолютная величина разности $\left(r_{i}\right)$ тормозного пути $S\left(V_{i}\right)$, полученного по уравнению (1), и тормозного пути, измеренного в процессе испытаний $\left(S_{i}\right)$ при скорости $\left(V_{i}\right)$ [2]:

$$
r_{i}=\operatorname{abs}\left(S\left(V_{i}\right)-S_{i}\right)
$$

Для выборки небольшого объема $(\mathrm{n}<25)$ используется метод вычисления максимального относительного отклонения [2]:

$$
\left|r_{i}-\bar{r}\right| / \bar{S} \leq \tau_{1-p}
$$

где $r_{i}-$ крайний (наибольший или наименьший) элемент разницы, вычисленный по формуле (12);

$\bar{r}$ - среднее значение отклонения;

$\bar{S}$ - среднеквадратическое отклонение;

$\tau_{1-p}-$ табличное значение статистики

$\tau$, вычисленной при доверительной вероятности 0,95 .

После исключения $i$-го значения вновь строится теоретическая кривая по сокращённой выборке и процесс повторяется до выполнения неравенства (12).

Для выборки большого объема используются таблицы распределения Стьюдента. Критическое значение $\tau_{p}$ ( $p$ процентная точка нормированного выборочного отклонения) выражается через критическое значение распределения Стьюдента ( $\left.\tau_{p, n-2}\right)$ по формуле [2]

$$
\tau_{(p, n)}=\frac{t_{(p, n-2)} \cdot \sqrt{n-1}}{\sqrt{n-2+\left(t_{(p, n-2)}\right)^{2}}} .
$$

Оценка параметров модели проверяется на значимость отличия от нуля по значимости коэффициента корреляции с использованием критерия Стьюдента. Расчетное значение критерия определяется по формуле [2] 


$$
\begin{aligned}
& t_{k p}=\frac{r_{x y} \cdot \sqrt{n-2}}{\sqrt{1-r_{x y}^{2}}}, \\
& r_{x y}=\frac{1}{(n-1) \cdot \sqrt{\sigma_{S}} \cdot \sqrt{\sigma_{V}}}\left(\sum_{i=1}^{n} S_{i} \cdot V_{i}-n \cdot \bar{S} \cdot \bar{V}\right),
\end{aligned}
$$

где $r_{x y}$ - коэффициент корреляции между

$S$ и $V$, который определяется по формуле

где $\sigma$ - дисперсия.

Табличное значение определяется по таблице $t$-распределения при значимости $\alpha=0,05$ и числе степеней свободы $k=n-2$.

Скорость движения поезда определялась путем экстраполяции фактической линейной скорости вращения колесной пары линейной зависимостью, причем в качестве опорных принимались локальные максимумы линейной скорости вращения колеса.

Особенности вращения колесной пары при срабатывании противоюзного устройства для различных скоростей в начале торможения представлены на рис. 1-4.

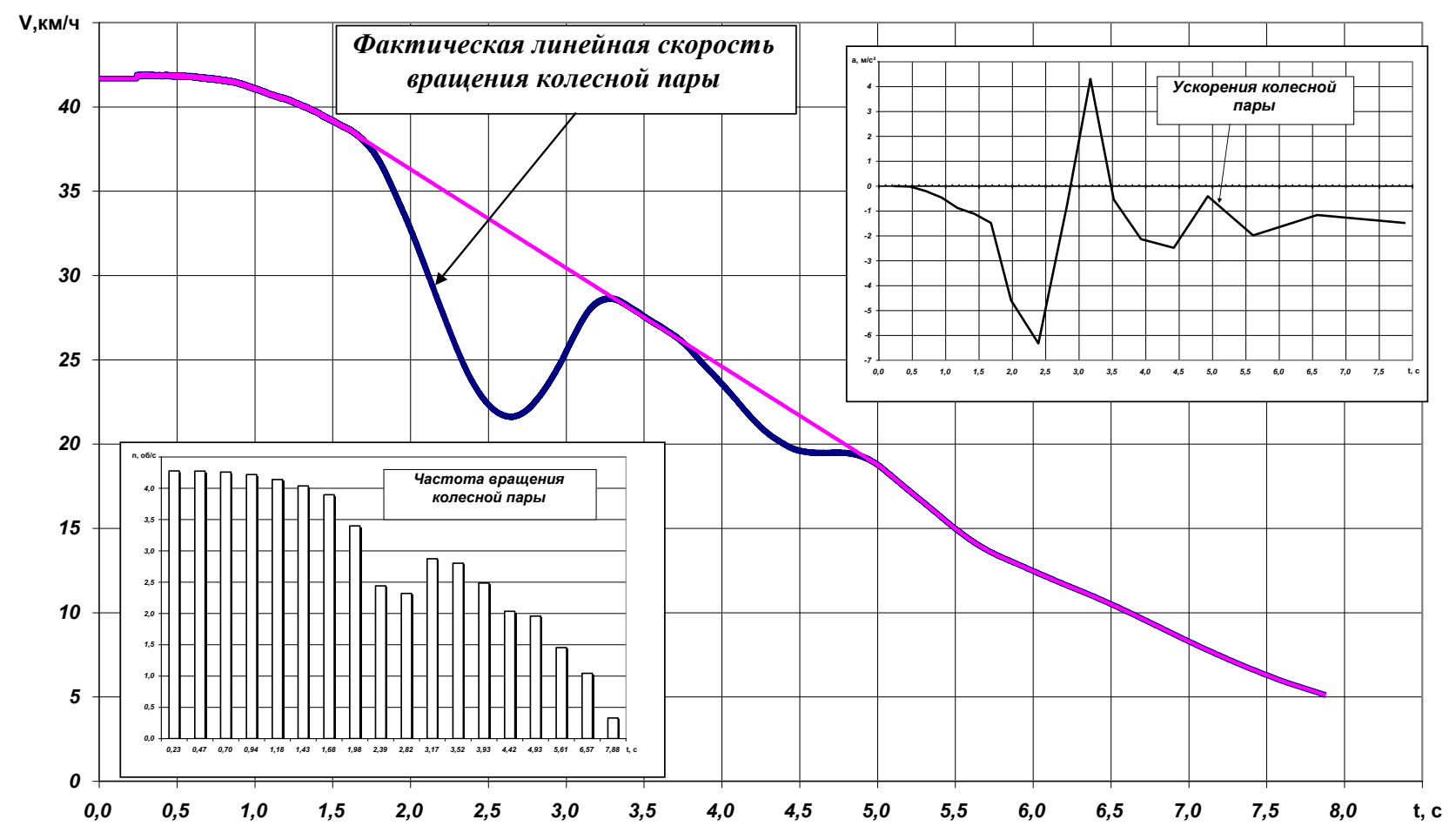

Рис. 1. Кинематика движения колесной пары при юзовой ситуации и скорости в начале торможения 41,6 км/ч 


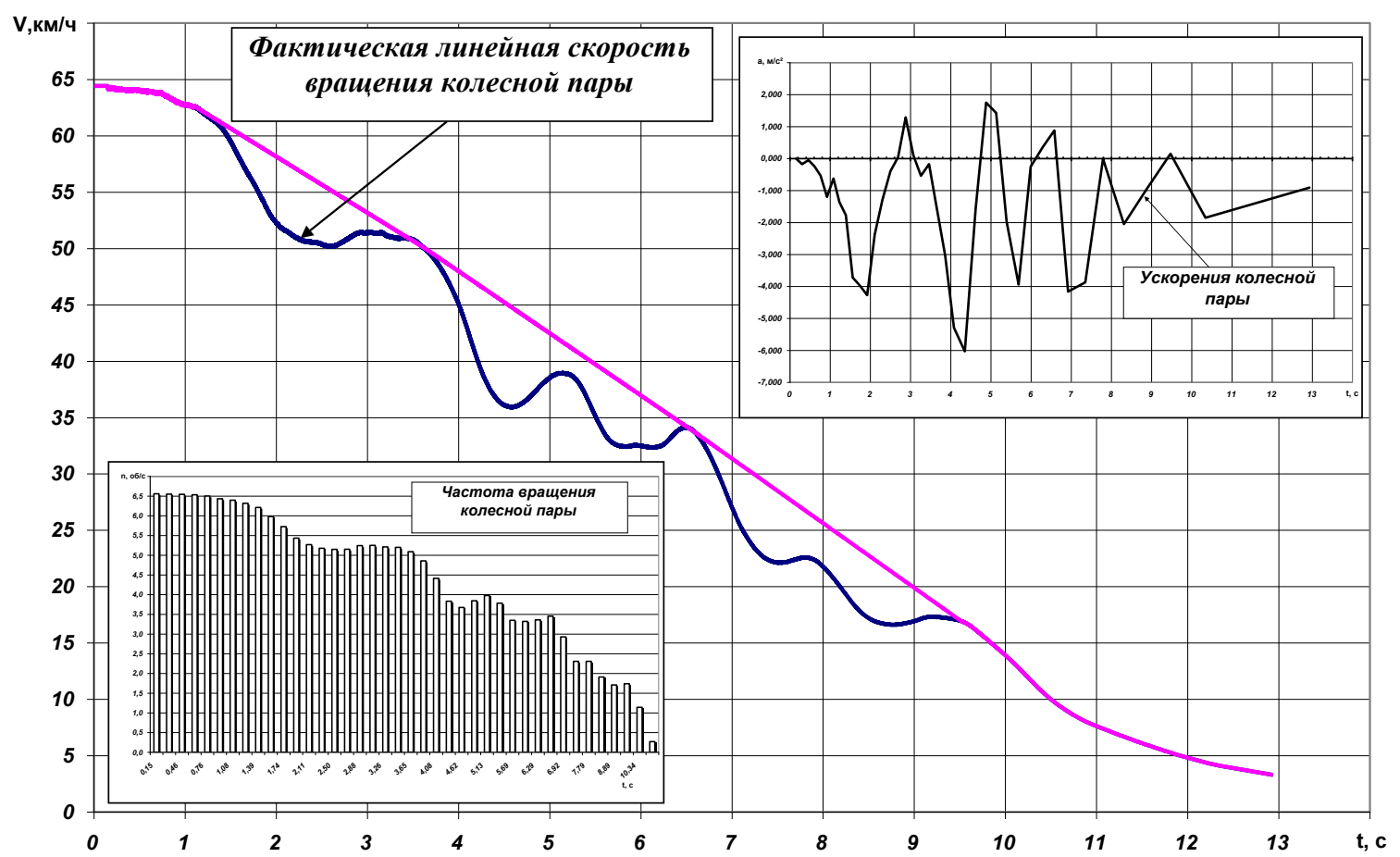

Рис. 2. Кинематика движения колесной пары при юзовой ситуации и скорости в начале торможения 63,79 км/ч

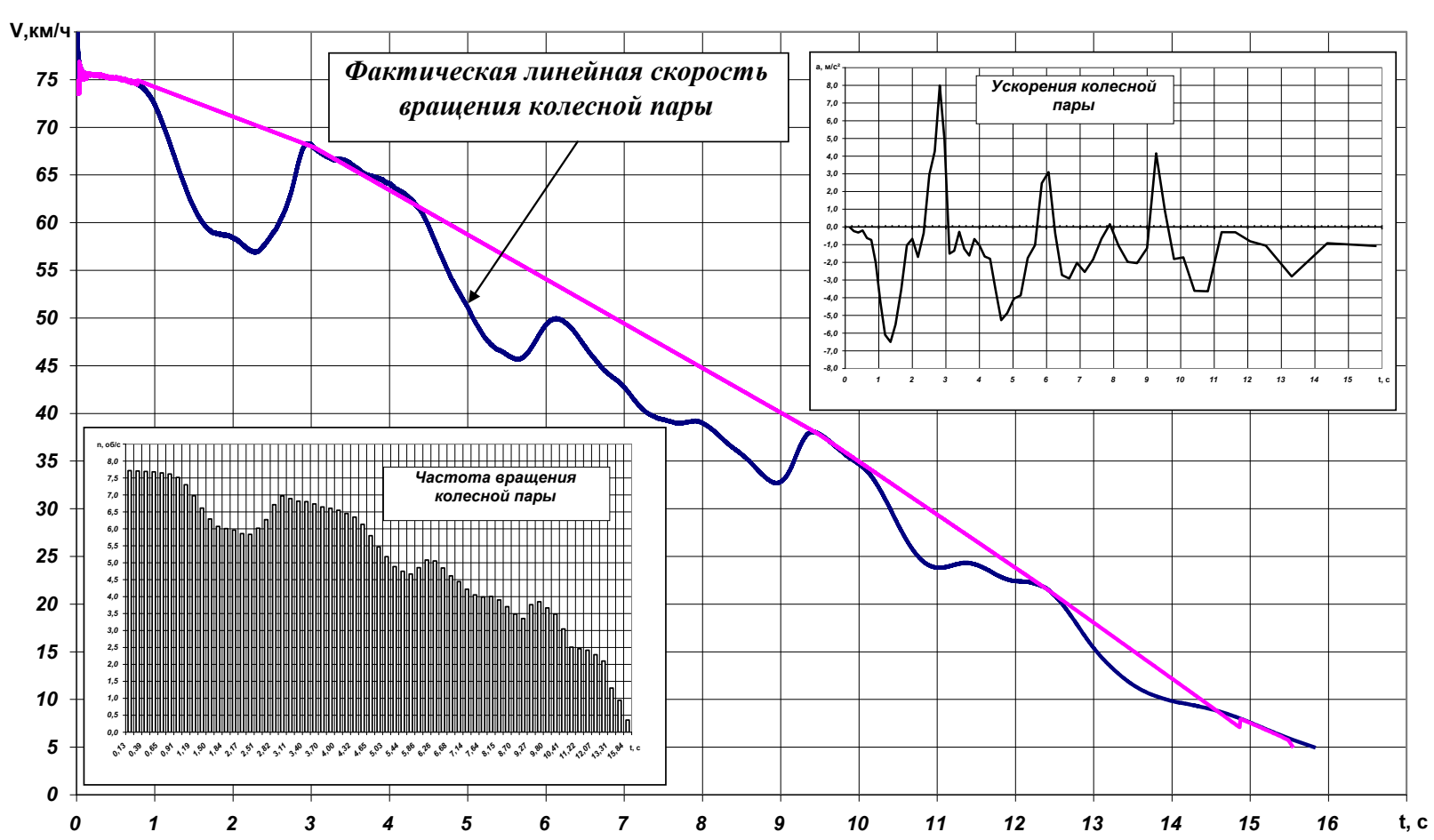

Рис. 3. Кинематика движения колесной пары при юзовой ситуации и скорости в начале торможения 75,08 км/ч 


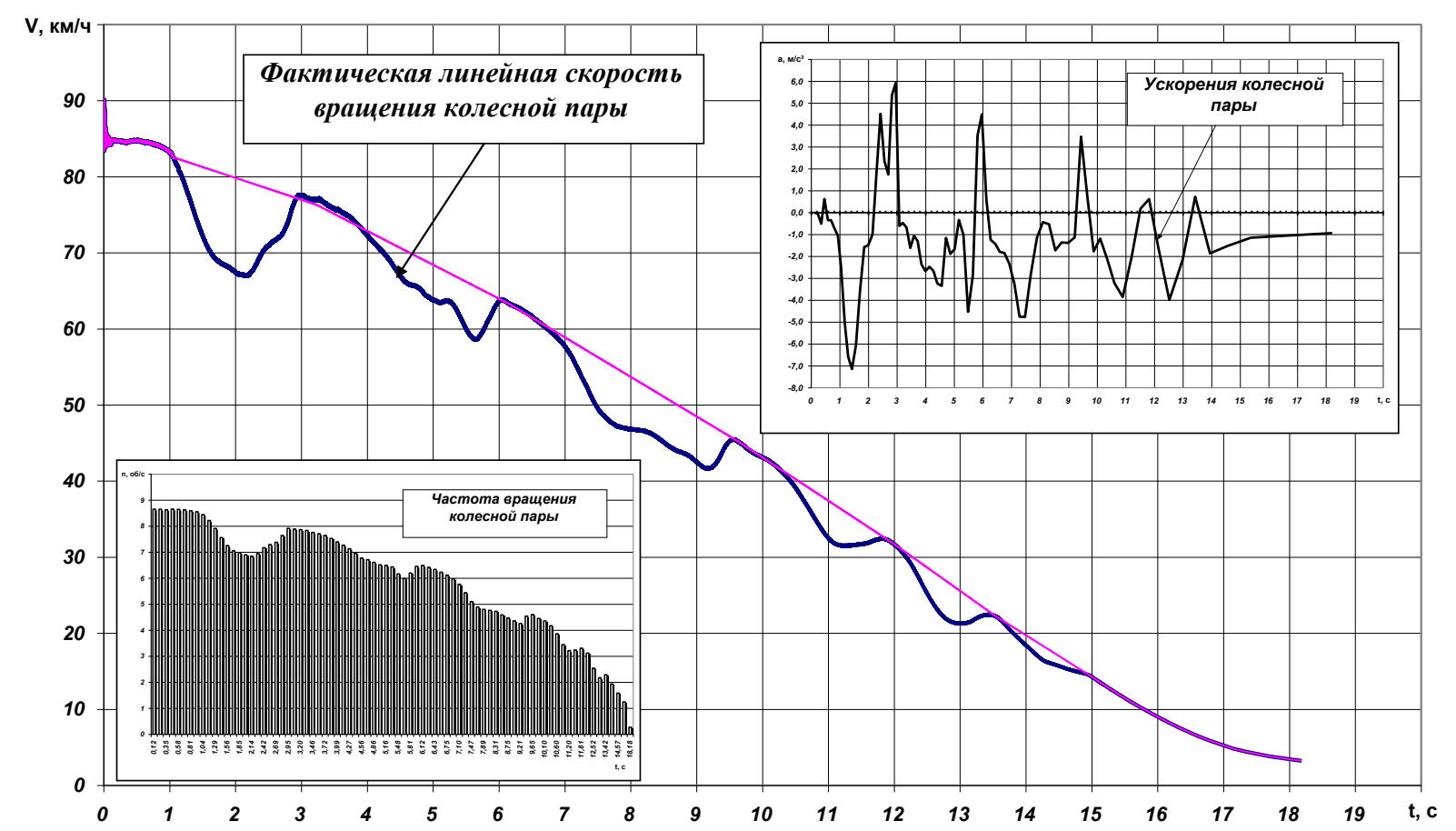

Рис. 4. Кинематика движения колесной пары при юзовой ситуации и скорости в начале торможения 84 км/ч

Выполненные расчетные исследования тормозных путей по вышеизложенной методике и сравнительный анализ показы- вают, что тормозные пути при срабатывании противоюзного устройства выше, чем при безъюзовом торможении (рис. 5).

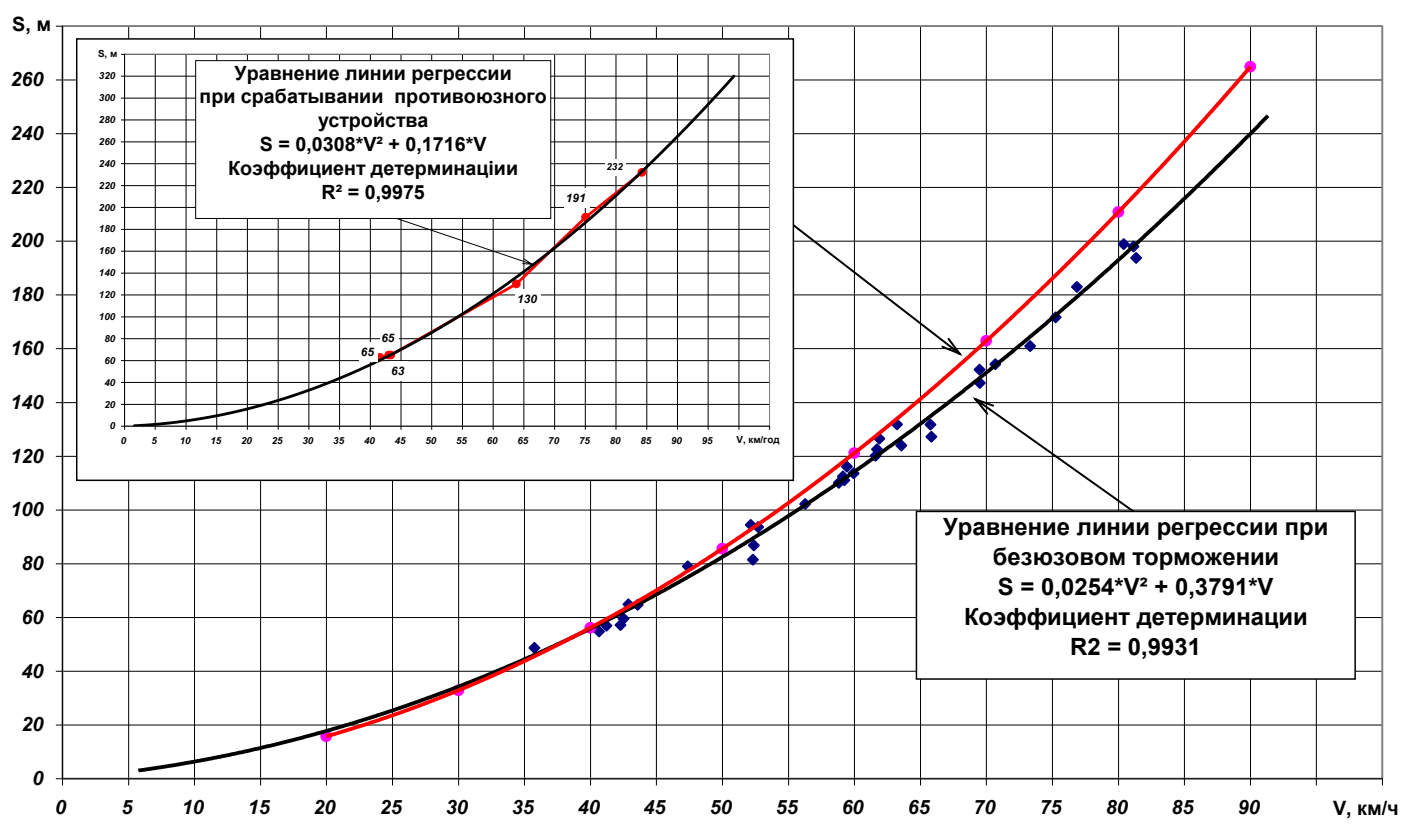

Рис. 5. Тормозные пути поезда метро при срабатывании противоюзного устройства и безъюзовом торможении на площадке 
На основе экспериментальных и расчетных исследований тормозной эффективности поезда метро при срабатывании противоюзного устройства установлено:

1. Тормозные пути поезда метро с ростом скорости в начале торможения увеличиваются, максимальное увеличение тормозного пути составляет $10 \%$ для скорости 90 км/ч (рис. 6).
2. Для скорости торможения менее $40 \mathrm{\kappa м} / ч$ противоюзное устройство не оказывает влияния на тормозной путь.

3. Тормозные пути поезда метро при экстренном пневматическом торможении на площадке в случае срабатывания противоюзного устройства не превышают допустимые значения тормозных путей (рис. 7).

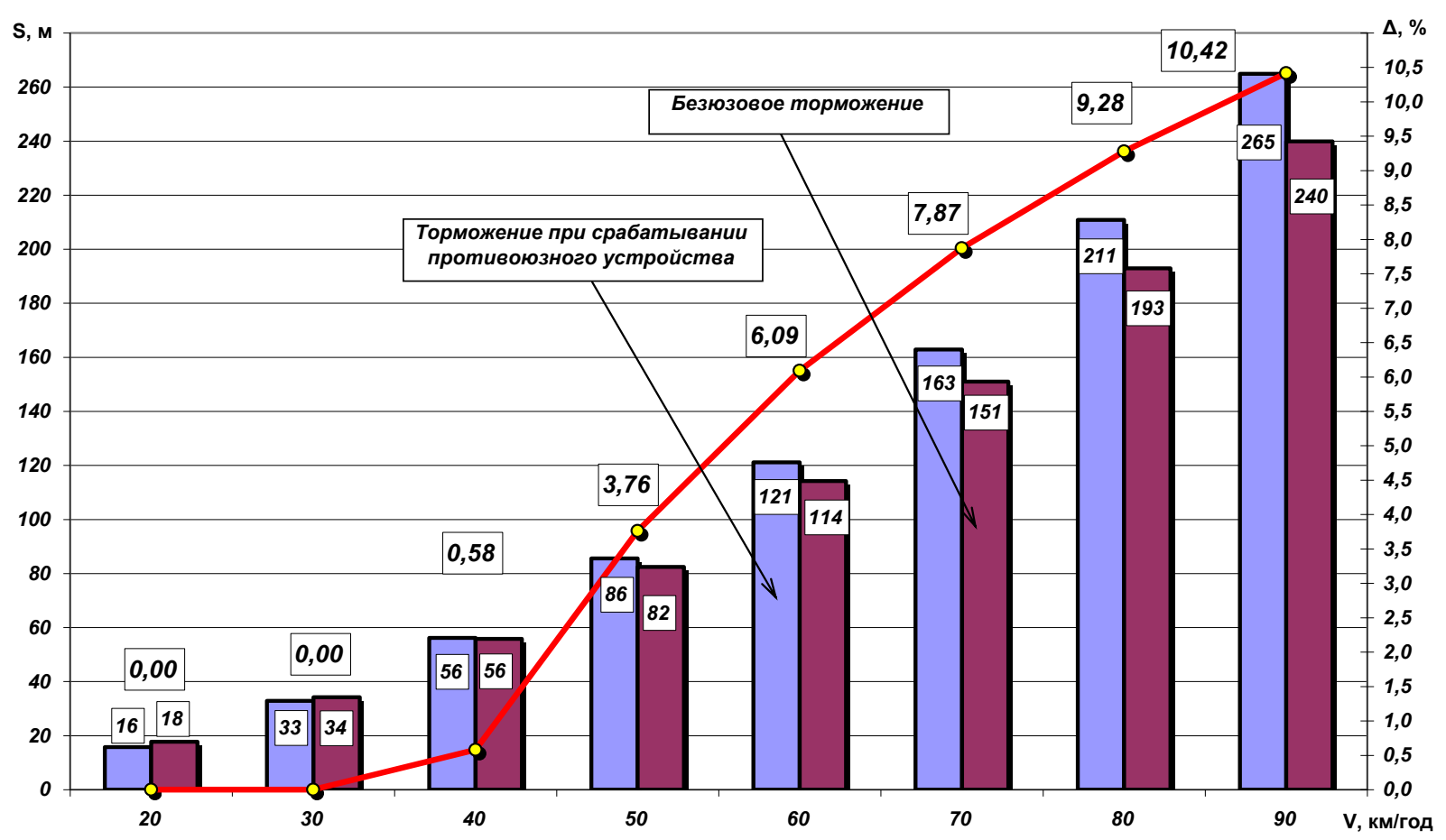

Рис. 6. Сравнительный анализ тормозных путей поезда метро на площадке 


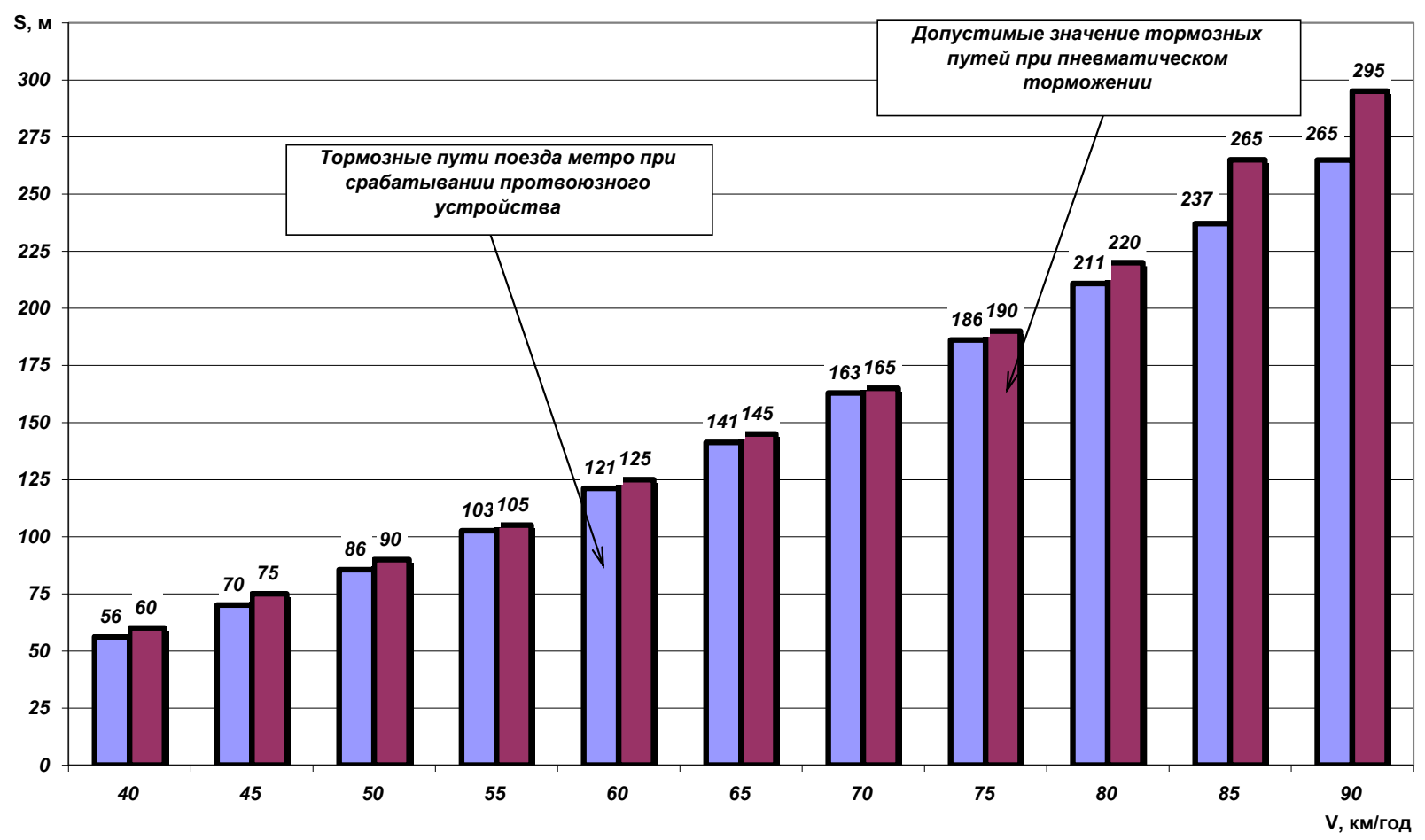

Рис. 7. Допустимые значения тормозных путей поезда метро при экстренном пневматическом торможении на площадке

\section{Список литературы}

1. Степанов, М.Н. Статистические методы обработки результатов механических испытаний [Текст]: справочник / М.Н. Степанов. - М.: Машиностроение, 1985.

2. Львовский, Э.Н. Статистические методы построения эмпирических формул [Текст] / Э.Н. Львовский. - М.: Высш. шк., 1988.

Ключевые слова: поезд метро, дисковый тормоз, противоюзное устройство, тормозной путь, скорость, частота вращения, коэффициент сцепления колеса с рельсом.

\section{Аннотации}

Наведені результати дослідження гальмівної ефективності поїзда метро при спрацьовуванні протиюзового пристрою. Показано, що гальмівні шляхи поїзда метро при екстреному пневматичному гальмуванні на площадці у разі спрацьовування протиюзового пристрою більше, ніж при без'юзовому гальмуванні. Перевищення тим більше, чим вища швидкість на початку гальмування і варіюється в межах від 0,58 \% для швидкості 40 км/год до 10,42 \% для швидкості 90 км/год.

Приведены результаты исследования тормозной эффективности поезда метро при срабатывании противоюзного устройства. Показано, что тормозные пути поезда метро при экстренном пневматическом торможении на площадке в случае срабатывания противоюзного устройства больше, чем при безъюзовом торможении. Превышение тем больше, чем выше скорость в начале торможения и варьируется в пределах от 0,58 \% для скорости 40 км/ч до 10,42 \% для скорости 90 км/ч. 
Research results of a metropolitan train braking efficiency at the antiskid device operation are given. It is shown that braking distances of the metropolitan train at emergency pneumatic braking on a level when the antiskid device is activated are longer than at antiskid braking. Exceeding is greater when initial braking speed rises and vary in the range from $0,58 \%$ for $40 \mathrm{~km} / \mathrm{h}$ speed to $10,42 \%$ for $90 \mathrm{~km} / \mathrm{h}$ speed. 